\title{
A randomized double-blind controlled trial of convalescent plasma in adults with severe COVID-19
}

\begin{abstract}
Max R. O'Donnell, ${ }^{1,2,3}$ Beatriz Grinsztejn, ${ }^{4}$ Matthew J. Cummings, ${ }^{1,3}$ Jessica E. Justman, ${ }^{2,5,6}$ Matthew R. Lamb, ${ }^{2,5}$ Christina M. Eckhardt, ${ }^{1}$ Neena M. Philip, ${ }^{5}$ Ying Kuen Cheung, ${ }^{7}$ Vinay Gupta, ${ }^{8}$ Esau João, ${ }^{9}$ Jose Henrique Pilotto, ${ }^{10}$ Maria Pia Diniz, ${ }^{4}$ Sandra Wagner Cardoso, ${ }^{4}$ Darryl Abrams, ${ }^{1}$ Kartik N. Rajagopalan, ${ }^{1}$ Sarah E. Borden, ${ }^{1}$ Allison Wolf, ${ }^{1}$ Leon Claude Sidi, ${ }^{9}$ Alexandre Vizzoni,, ${ }^{4}$ Valdilea G. Veloso, ${ }^{4}$ Zachary C. Bitan, ${ }^{11}$ Dawn E. Scotto, ${ }^{1}$ Benjamin J. Meyer, ${ }^{12}$ Samuel D. Jacobson, ${ }^{12}$ Alex Kantor, ${ }^{1}$ Nischay Mishra, ${ }^{3}$ Lokendra V. Chauhan, ${ }^{3}$ Elizabeth F. Stone, ${ }^{11}$ Flavia Dei Zotti, ${ }^{11}$ Francesca La Carpia, ${ }^{11}$ Krystalyn E. Hudson, ${ }^{11}$ Stephen A. Ferrara, ${ }^{11}$ Joseph Schwartz, ${ }^{11}$ Brie A. Stotler, ${ }^{11}$ Wen-Hsuan W. Lin, ${ }^{11}$ Sandeep N. Wontakal, ${ }^{11}$ Beth Shaz, ${ }^{13}$ Thomas Briese, ${ }^{3}$ Eldad A. Hod, ${ }^{11}$ Steven L. Spitalnik, ${ }^{11}$ Andrew Eisenberger, ${ }^{14}$ and Walter I. Lipkin ${ }^{2,3,11}$

'Division of Pulmonary, Allergy, and Critical Care Medicine, Department of Medicine, Columbia University Irving Medical Center, New York, New York, USA. ${ }^{2}$ Department of Epidemiology, and ${ }^{3}$ Center for Infection and Immunity, Columbia University Mailman School of Public Health, New York, New York, USA. ${ }^{4}$ Instituto Nacional de Infectologia Evandro Chagas-Fiocruz, Rio de Janeiro, Brazil. ${ }^{I}$ ICAP, Columbia University Mailman School of Public Health, New York, New York, USA. ${ }^{6}$ Division of Infectious Diseases, Department of Medicine, Columbia University Irving Medical Center, New York, New York, USA. 'Department of Biostatistics, Columbia University Mailman School of Public Health, New York, New York, USA. ${ }^{8}$ Institute for Health Metrics and Evaluation, University of Washington, Seattle, Washington, USA. ${ }^{9}$ Hospital Federal dos Servidores do Estado, Rio de Janeiro, Brazil. ${ }^{10}$ Hospital Geral de Nova Iguaçu, Rio de Janeiro, Brazil and Laboratório de Aids e Imunologia Molecular, Instituto Oswaldo Cruz - Fiocruz, Rio de Janeiro, Brazil. "Department of Pathology and Cell Biology, Columbia University Irving Medical Center, New York, New York, USA. ${ }^{2}$ Vagelos College of Physicians and Surgeons, Columbia University, New York, New York, USA. ${ }^{13}$ New York Blood Center, New York, New York, USA. ${ }^{14}$ Division of Hematology and Oncology, Department of Medicine, Columbia University Irving Medical Center, New York, New York, USA.
\end{abstract}

BACKGROUND. Although convalescent plasma has been widely used to treat severe coronavirus disease 2019 (COVID-19), data from randomized controlled trials that support its efficacy are limited.

METHODS. We conducted a randomized, double-blind, controlled trial among adults hospitalized with severe and critical COVID-19 at 5 sites in New York City (USA) and Rio de Janeiro (Brazil). Patients were randomized 2:1 to receive a single transfusion of either convalescent plasma or normal control plasma. The primary outcome was clinical status at 28 days following randomization, measured using an ordinal scale and analyzed using a proportional odds model in the intention-to-treat population.

RESULTS. Of 223 participants enrolled, 150 were randomized to receive convalescent plasma and 73 to receive normal control plasma. At 28 days, no significant improvement in the clinical scale was observed in participants randomized to convalescent plasma (OR 1.50, 95\% confidence interval [CI] 0.83-2.68, $P=\mathbf{0 . 1 8 0}$ ). However, 28-day mortality was significantly lower in participants randomized to convalescent plasma versus control plasma (19/150 [12.6\%] versus 18/73 [24.6\%], OR 0.44, 95\% CI 0.22-0.91, $P=0.034)$. The median titer of anti-SARS-CoV-2 neutralizing antibody in infused convalescent plasma units was 1:160 (IQR 1:80-1:320). In a subset of nasopharyngeal swab samples from Brazil that underwent genomic sequencing, no evidence of neutralization-escape mutants was detected.

CONCLUSION. In adults hospitalized with severe COVID-19, use of convalescent plasma was not associated with significant improvement in day 28 clinical status. However, convalescent plasma was associated with significantly improved survival. A possible explanation is that survivors remained hospitalized at their baseline clinical status.

TRIAL REGISTRATION. ClinicalTrials.gov, NCT04359810.

FUNDING. Amazon Foundation, Skoll Foundation.

Conflict of interest: MRO and MJC participated as investigators for clinical trials evaluating the efficacy and safety of remdesivir in hospitalized patients with COVID-19, sponsored by Gilead Sciences. VG is employed by Amazon Care. Copyright: (5) 2021, American Society for Clinical Investigation. Submitted: April 20, 2021; Accepted: May 6, 2021; Published: July 1, 2021 Reference information: J Clin Invest. 2021;131(13):e150646. https://doi.org/10.1172/JCl150646.

\section{Introduction}

As of April 19, 2021, over 141 million cases of coronavirus disease 2019 (COVID-19) associated with severe acute respiratory syndrome coronavirus-2 (SARS-CoV-2) had been reported worldwide (1). Available data suggest that approximately $10 \%-$ $25 \%$ of patients with SARS-CoV-2 infection develop severe COVID-19 characterized primarily by pneumonia and in a sub- 


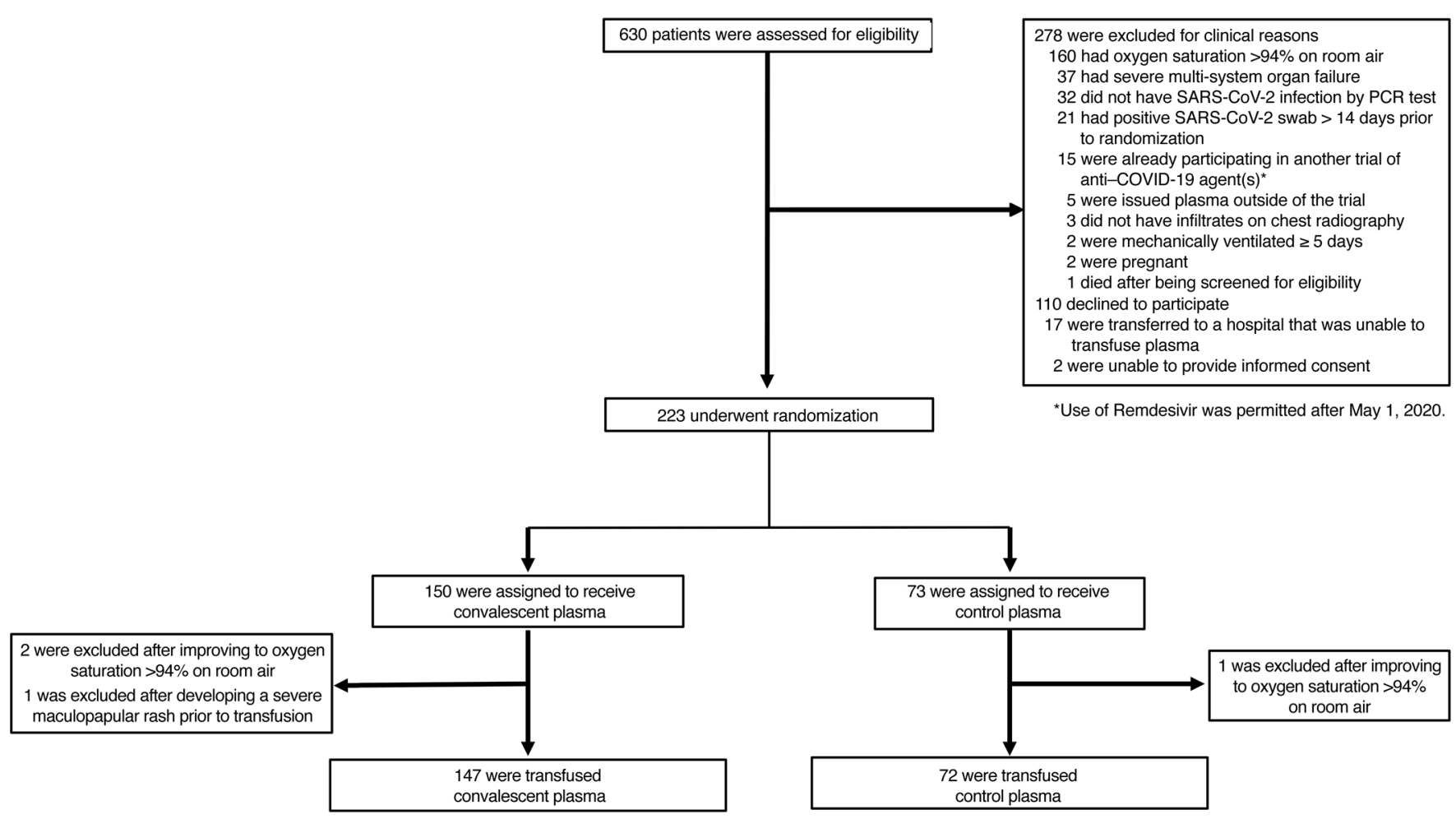

Figure 1. Trial flow diagram.

set, acute respiratory distress syndrome (ARDS) (2-4). Among severe cases, mortality occurs in 39\%-49\% $(2,4)$.

Following the emergence of SARS-CoV-2, convalescent plasma was proposed as a rapidly scalable therapeutic to prevent or mitigate severe illness through virus neutralization or antibody-dependent immunomodulation (5). During recent epidemics of emerging respiratory viruses such as SARS-CoV, H5N1, and 2009 H1N1 influenza, observational and nonrandomized studies reported improved clinical outcomes and minimal adverse effects associated with use of convalescent plasma in severely ill patients (6). In patients with severe COVID-19, observational studies have suggested possible clinical efficacy and safety using convalescent plasma, primarily among patients not receiving invasive mechanical ventilation (IMV) and those with shorter durations of illness (7-10). Despite these signals, data from randomized controlled trials supporting use of convalescent plasma in hospitalized patients with COVID-19 are limited. Open-label trials, including the large Randomised Evaluation of COVID-19 Therapy (RECOVERY) trial, reported no significant improvements in clinical outcomes among patients hospitalized with severe COVID-19 (11-13). A double-blind, placebo-controlled trial in Argentina also reported no improvement in clinical outcomes with use of convalescent plasma among adults hospitalized with severe COVID-19, including among subgroups stratified by illness duration and clinical severity (14).

In the United States and Brazil, approximately 31 and 14 million cases of COVID-19 have been reported as of April 19, 2021, respectively (1). Given the lack of effective medical therapies against SARS-CoV-2, we conducted a randomized, double-blind, controlled phase 2 clinical trial to evaluate the clin- ical efficacy and safety of convalescent plasma among adults hospitalized with severe and critical COVID-19 in New York City and Rio de Janeiro.

\section{Results}

Participants. Between April 21 and November 27, 2020, a total of 630 patients were evaluated for inclusion criteria across the 5 study sites. Two-hundred twenty-three were enrolled, randomized, and included in the intention to treat (ITT) analysis (Figure 1). Four participants were randomized but did not receive their assigned treatment: 3 participants ( 2 randomized to convalescent plasma and 1 to control plasma) had improvements in oxygen saturation to greater than $94 \%$ prior to transfusion, and 1 participant randomized to convalescent plasma developed a maculopapular rash prior to receipt of plasma, for which subsequent transfusion was deferred. Thus, 219 patients were included in the per-protocol and safety analysis: 147 participants transfused convalescent plasma, and 72 participants transfused control plasma (Figure 1). Data on neutralizing antibody titers were available for $89 \%$ (130/150) of convalescent plasma units. Of these, the median titer was 1:160 (IQR 1:80-1:320).

Of the 223 participants enrolled, 73 were enrolled in New York City and 150 in Rio de Janeiro (Table 1). The median age of participants was 61 years and 66\% (147/223) were male. The median duration of symptoms prior to randomization was 9 days. Nearly all participants required respiratory support at baseline: $57 \%(126 / 223)$ of participants required supplemental oxygen, 25\% (55/223) required high-flow oxygen therapy or noninvasive mechanical ventilation, and $13 \%(28 / 223)$ required IMV or ECMO. Some imbalances were present between treatment groups. Participants enrolled in the convalescent plasma group were younger, with fewer men and a slight- 


\section{Table 1. Baseline patient characteristics}

\begin{tabular}{lcc} 
Variable & $\begin{array}{c}\text { Convalescent plasma } \\
\boldsymbol{N}=150\end{array}$ & $\begin{array}{c}\text { Normal control plasma } \\
\boldsymbol{N}=73\end{array}$ \\
$\begin{array}{l}\text { Sex, } \boldsymbol{n}(\%) \\
\text { Male }\end{array}$ & $96(64)$ & $51(70)$ \\
\hline Female & $54(36)$ & $22(30)$ \\
$\quad$ Age in years, median (IQR) & $60(48-71)$ & $63(49-72)$ \\
$\begin{array}{l}\text { Age group, } \boldsymbol{n}(\%) \\
<60 \text { years }\end{array}$ & $28(38)$ \\
$60-69$ years & $74(49)$ & $24(33)$ \\
$70-79$ years & $35(23)$ & $16(22)$ \\
$\geq 80$ years & $28(19)$ & $5(7)$ \\
$\begin{array}{l}\text { Ceographic location } \\
\text { United States } \\
\text { Brazil }\end{array}$ & $13(9)$ & $24(33)$ \\
\hline
\end{tabular}

Body mass index

BMI, median (IQR)

$\mathrm{BMI} \geq 30 \mathrm{~kg} / \mathrm{m}^{2}$

$30.1(26.6-34.7)$

76 (51)

$29.4(26.2-33.0)$

Baseline conditions, $n$ (\%)

Hypertension

Diabetes mellitus

Chronic cardiac disease

$53(35)$

$55(37)$

56 (37)

$33(45)$

Chronic kidney disease

13 (9)

15 (10)

Chronic pulmonary disease

3 (2)

nic liver disease

4 (3)

Hyperlipidemia

27 (18)

$22(30)$

Duration of COVID-19 symptoms prior

to randomization, days, median (IQRR) ${ }^{\mathrm{B}}$

$10(7-13)$

27 (37)

$28(38)$

8 (11)

5 (7)

$1(1)$

0 (0)

$9(12)$

ymptoms reported, $n$ (\%)

$\begin{array}{lll}\text { Shortness of breath } & 125(83) & 58(79) \\ \text { Fever } & 66(44) & 27(37) \\ \text { Cough } & 114(76) & 49(67)\end{array}$

Clinical status at randomization based on ordinal scale ${ }^{\complement}$

$\begin{array}{lcc}\begin{array}{l}\text { 3: Hospitalized, not requiring } \\ \text { supplemental oxygen }\end{array} & 5(3) & 5(7) \\ \begin{array}{l}\text { 4-5: Hospitalized, requiring } \\ \text { supplemental oxygen, HFO, NIV }\end{array} & 125(83) & 57(78) \\ \begin{array}{l}\text { 6: Hospitalized, requiring IMV, } \\ \text { ECMO, or both }\end{array} & 17(11) & 11(15)\end{array}$

Concomitant medications received during study period

$\begin{array}{lcc}\text { Corticosteroids } & 121(81) & 60(82) \\ \text { Remdesivir } & 8(5) & 5(7) \\ \text { Hydroxychloroquine } & 8(5) & 5(7) \\ \text { Antibacterial agent } & 111(74) & 60(82)\end{array}$

AUnknown for 4 patients ( 2 in each treatment group). ${ }^{B}$ Unknown for 6 patients (3 in each treatment group). 'Baseline clinical status unknown for for 3 patients (all inconvalescent plasma group).

ly longer symptom duration. During the trial period, $81 \%(181 / 223)$ of participants received corticosteroids and 6\% (13/223) received remdesivir, the latter exclusively in New York City.

Primary outcome assessment of clinical status at 28 days was completed for 215 (96\%) of 223 randomized patients. Eight participants with indeterminate clinical status at day 28 were discharged alive but were unable to be contacted at day 28 . Of these 8 participants, 3 had at least 14 days of follow-up and 5 had less than 14 days of follow-up.
Primary outcome. Using a 1-sided Mann-Whitney test of the alternative hypothesis favoring the convalescent plasma arm, the primary outcome analysis of the ITT population was consistent with a "go" decision $(P=0.09)$. Although participants randomized to receive convalescent plasma had 1.5 times the odds of a 1-point improvement in clinical status at day 28 , this difference was not statistically significant (OR 1.5, 95\% confidence interval [CI] 0.83-2.68, $P=$ 0.18) (Table 2). After adjustment for age, sex, and illness duration, the odds of improvement were similar (Table 2). Results were also similar in unadjusted and adjusted analyses of the per-protocol population and in 2 sensitivity analyses, one in which the 8 participants without a definitive day 28 outcome were considered deceased, and another in which the last available clinical status was carried forward for patients with at least 14 days of follow-up and patients with fewer than 14 days of follow-up were considered deceased (Supplemental Tables 1-3; supplemental material available online with this article; https://doi.org/10.1172/JCI150646DS1).

28-day mortality. In the ITT population, mortality at 28 days was significantly lower among participants randomized to convalescent versus control plasma (19/150 [12.6\%] versus 18/73 [24.6\%], OR $0.44,95 \%$ CI $0.22-0.91, P=0.034$ ) when the last available clinical status was carried forward for the 8 patients without definitive day 28 outcome status (Table 2 and Figure 2). These results were consistent in adjusted analyses and in sensitivity analyses to account for the 8 patients without definitive day 28 outcome (Table 2 and Supplemental Tables 4 and 5). All recorded deaths occurred during hospitalization. No significant between-group differences were observed in the other secondary outcomes (Table 2 and Figure 3).

Subgroup analyses. In prespecified analyses of the primary outcome based on respiratory support and symptom duration at baseline, no significant between-group differences were observed (Figure 4 and Supplemental Tables 6 and 7). However, we observed trends toward improved clinical status among patients who received convalescent plasma 7 days or earlier after symptom onset and those who received convalescent plasma with higher-titers of neutralizing antibody and concomitant corticosteroids (Figure 4, Supplemental Figures 1 and 2, and Supplemental Tables 6 and 7). The median time to corticosteroids administration was 1 day prior to transfusion in the convalescent plasma and control plasma intervention groups (IQR -2 to 0 days for both). In stratified analyses of 28-day mortality, unadjusted point estimates consistently favored the convalescent plasma group (Supplemental Figure 3).

SARS-CoV-2 genomic sequencing. RNA template was sufficient to recover near complete ( $>99 \%$ ) genomic sequence from 40 nasopharyngeal samples from Brazil. Twenty-nine (73\%) samples represented common clades circulating worldwide and had no spike protein mutations. None of the samples contained the mutations characteristic of B.1.1.28 P1. Four had mutations found in B.1.1.28 (E484K) but did not have the N501Y, K417N/T mutations found in P1. One sample had 3 of 4 mutations characteristic of B.1.1.28 (AM-II), including V1176K in S, that is not known to impair neutralization. In short, we found no evidence of neutralization-escape mutants.

Safety analysis. Serious adverse events occurred in 39 of 147 (26.5\%) patients who received convalescent plasma and 26 of 72 (36.1\%) patients who received control plasma (Supplemental Tables $8-11)$. Adverse events considered as definitely or probably associated with plasma transfusion were reported in 4 of 147 (2.7\%) patients 


\begin{tabular}{|c|c|c|c|c|c|c|}
\hline Outcomes & $\begin{array}{c}\text { Convalescent plasma } \\
\qquad N=150\end{array}$ & $\begin{array}{c}\text { Control plasma } \\
\qquad N=73\end{array}$ & $\begin{array}{l}\text { OR or SHR } \\
(95 \% \mathrm{Cl})\end{array}$ & $P$ value & $\begin{array}{c}\text { Adjusted OR or sHR } \\
(95 \% \mathrm{Cl})^{A}\end{array}$ & $P$ value \\
\hline Primary outcome, clinical status at 28 days, $n(\%)$ & & & OR $1.50(0.83-2.68)$ & 0.180 & OR $1.38(0.73-2.61)$ & 0.318 \\
\hline 1 and 2: Not hospitalized & $108(72.0)$ & $48(65.8)$ & & & & \\
\hline 3: Hospitalized, not requiring supplemental oxygen & $3(2.0)$ & $2(2.7)$ & & & & \\
\hline $\begin{array}{l}\text { 5: Hospitalized, requiring high-flow oxygen therapy } \\
\text { or noninvasive mechanical ventilation }\end{array}$ & $1(0.7)$ & $0(0.0)$ & & & & \\
\hline 6: Hospitalized, requiring IMV, ECMO, or both & $12(8.0)$ & $4(5.5)$ & & & & \\
\hline 7: Dead & $19(12.6)$ & $18(24.6)$ & & & & \\
\hline \multicolumn{7}{|l|}{ Secondary outcomes } \\
\hline Time to clinical improvement, median, days (IQR) $)^{\mathrm{B}}$ & $5(4-6)$ & $7(5-8)$ & sHR $1.21(0.89-1.65)$ & 0.231 & sHR $1.20(0.87-1.64)$ & 0.261 \\
\hline Time to hospital discharge, median, days (IQR) & $9(6-28)$ & $8(6-22)$ & SHR 1.05 (0.77-1.43) & 0.756 & SHR 1.02 (0.75-1.38) & 0.913 \\
\hline
\end{tabular}

${ }^{A}$ Adjusted for age (continuous variable), sex, and duration of symptoms at baseline (duration of symptoms unknown for 6 patients). ${ }^{B}$ Baseline outcome assessment unknown for 3 patients (all in treatment group). CNo patients were known to have died following discharge from hospital. ${ }^{\mathrm{D}}$ Thirteen patients were excluded from unadjusted analysis (10 participants enrolled but did not require supplemental oxygen, 3 patients without a baseline assessment) and 16 patients were excluded from adjusted analysis. SHR, subhazard ratio.

who received convalescent plasma and 3 of $72(4.2 \%)$ patients who received control plasma. In patients who received convalescent plasma, these events included worsening anemia, urticaria, skin rash, and transfusion-associated circulatory overload.

\section{Discussion}

In this randomized, blinded, and controlled phase 2 trial conducted in New York City and Rio de Janeiro, treatment with convalescent plasma as compared with control plasma did not result in significant clinical improvement at 28 days, based on an ordinal scale of clinical status, among adults hospitalized with severe and critical COVID-19. However, mortality at 28 days was significantly lower among patients randomized to convalescent plasma. This effect was observed across analyses adjusted for imbalances in baseline variables with prognostic relevance and in sensitivity analyses performed to account for indeterminate 28-day vital status in 8 patients.

Although limited, available data suggest that treatment efficacy for convalescent plasma may be dependent on illness duration and severity and titers of neutralizing anti-SARS-CoV-2 antibody in transfused plasma. In a recent clinical trial from Argentina, transfusion of high-titer convalescent plasma within 72 hours of symptom onset prevented progression to severe illness among elderly adults with mild COVID-19 (15). In contrast, no overall improvements in clinical status were observed in recent trials of convalescent plasma among inpatients with severe COVID-19 in China, Argentina, and the United Kingdom (11-13). However, subgroup analyses in 2 of these trials suggested a possible benefit among patients with less severe and shorter durations of illness $(11,12)$. These signals are consistent with results of a retrospective study of more than 3000 U.S. adults who received convalescent plasma for treatment of severe COVID-19 (10). In this analysis, high-titer convalescent plasma was associated with improved mortality among inpatients who were not receiving IMV at the time of transfusion. Considering power limitations of our trial, we similarly observed trends toward improvement in the primary outcome among patients in the convalescent plasma group who were transfused within 7 days of symptom onset and those who received convalescent plasma with higher titers of neutralizing anti-SARS-CoV-2 antibody.

In the context of emerging SARS-CoV-2 variants, some of which may be associated with greater transmissibility and more severe illness (16), convalescent plasma may offer distinct therapeutic advantages. Since convalescent plasma, which contains polyclonal antibodies, may be donated and transfused locally, its use may be more adaptable to rapidly changing local viral ecology than other interventions. In contrast, monoclonal antibody therapies may need to be repeatedly engineered and combined to optimize potency among emergent SARS-CoV-2 variants $(17,18)$. Further, since collection and distribution of convalescent plasma units can be performed using existing blood donation protocols and infrastructure, convalescent plasma may be more scalable for use in low- and middle-income countries.

Mortality at 28 days was significantly lower among patients randomized to receive convalescent plasma. This important secondary finding contrasts with our primary outcome, which shows no significant difference in clinical scale through day 28. One possible explanation for this apparent contradiction may be that although patients randomized to convalescent plasma had higher odds for survival, they remained hospitalized at their baseline clinical status (e.g., mechanically ventilated) and therefore did not achieve an improvement in 28-day clinical score (4). Although 


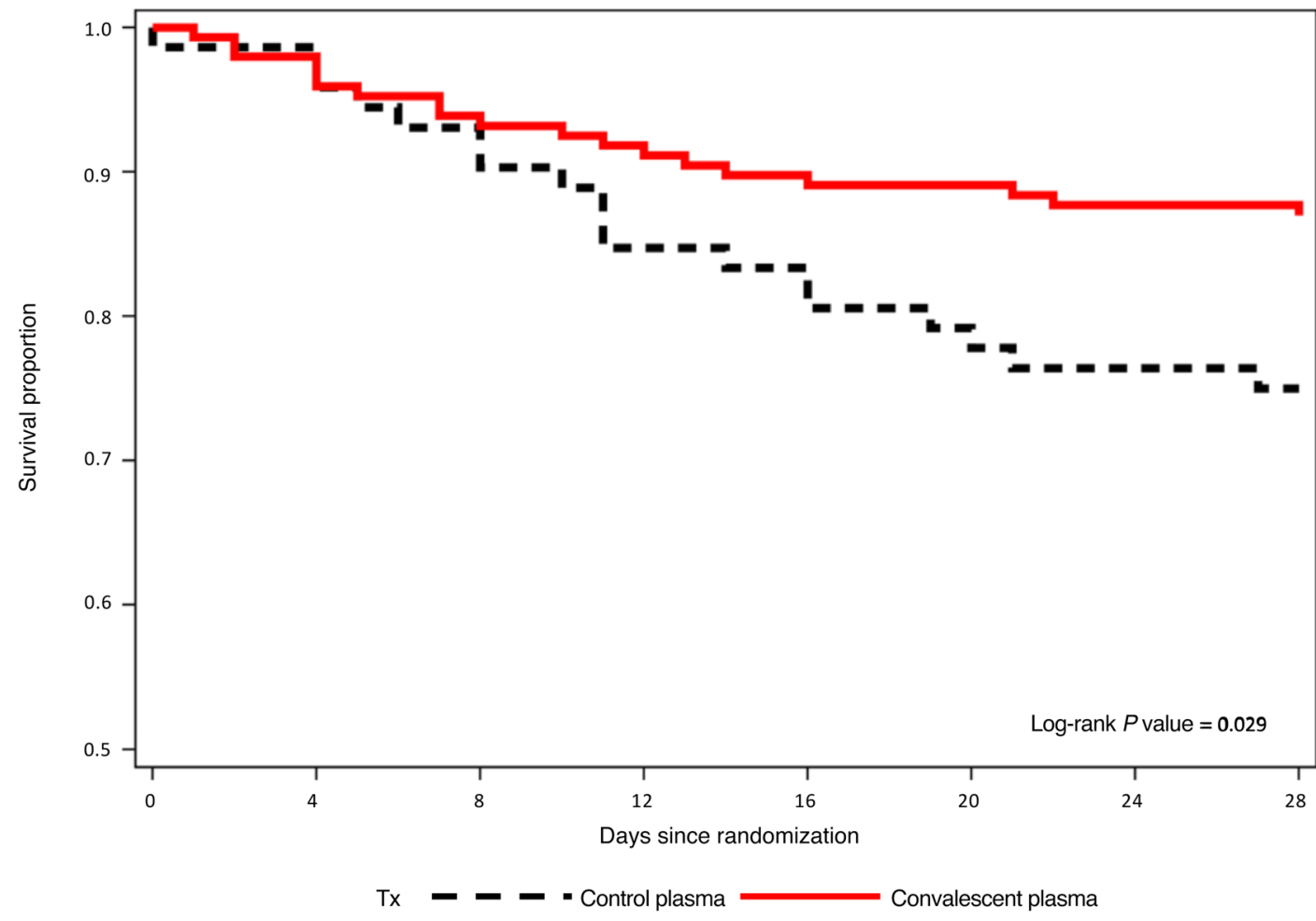

\begin{tabular}{|c|c|c|c|c|c|c|c|c|c|}
\hline \multicolumn{2}{|c|}{ Time since randomization (days) } & 0 & 4 & 8 & 12 & 16 & 20 & 24 & 28 \\
\hline & At risk & 73 & 71 & 67 & 61 & 60 & 57 & 55 & 50 \\
\hline & At risk & 150 & 146 & 137 & 134 & 131 & 139 & 128 & 122 \\
\hline
\end{tabular}

Figure 2. Kaplan-Meier estimates of mortality, stratified by treatment group.

this secondary outcome was prespecified, our study was not powered to detect a difference in 28-day mortality, and analyses of our secondary outcomes were not adjusted for multiplicity. This finding should be interpreted with caution as it differs from results of larger inpatient trials adequately powered to detect differences in mortality, such as RECOVERY (13).

We observed no significant difference in adverse events between treatment groups and very few events were considered related to plasma infusion. Although use of control plasma may have potentially contributed to hypercoagulability (19), the incidence of thrombotic events in our study population was similar to that reported in observational studies of patients with severe COVID-19 (20).

Our trial has several strengths. First, the randomized, blinded, controlled design of our trial was implemented with high adherence to the study protocol. Second, we enrolled severe and critical COVID-19 patients in racially and ethnically diverse urban settings in 2 countries. Third, our strategy for qualification and collection of convalescent plasma was pragmatic, increasing generalizability of our findings to settings where quantification of neutralization activity is unavailable. However, we quantified neutralizing antibody titers in approximately $90 \%$ of convalescent plasma samples post hoc. Fourth, our use of control plasma was a strength since both study agents had the same appearance, enhancing the blinded nature of the trial, and both had a similar effect on volume expansion. As convalescent plasma may have other immunomodulatory factors apart from anti-SARS-CoV-2 antibodies, such as immunoglobulins, hemostatic proteins, and cytokines, use of normal plasma as a comparator allowed us to evaluate the effect of convalescent antibodies while controlling for these other factors.

Our trial has several limitations. Although convalescent plasma was collected from donors with anti-SARS-CoV-2 total IgG antibody titer of at least 1:400, neutralizing antibody titers in some convalescent plasma units were low, and we do not have data on antibody titers in patient samples before and after transfusion. Second, although all control plasma units were collected prior to the first known cases of COVID-19 in Rio de Janeiro and New York City, 1 out of 19 units tested neutralized SARS-CoV-2 at low titer. Although this could represent a false-positive, it is possible that oth- 


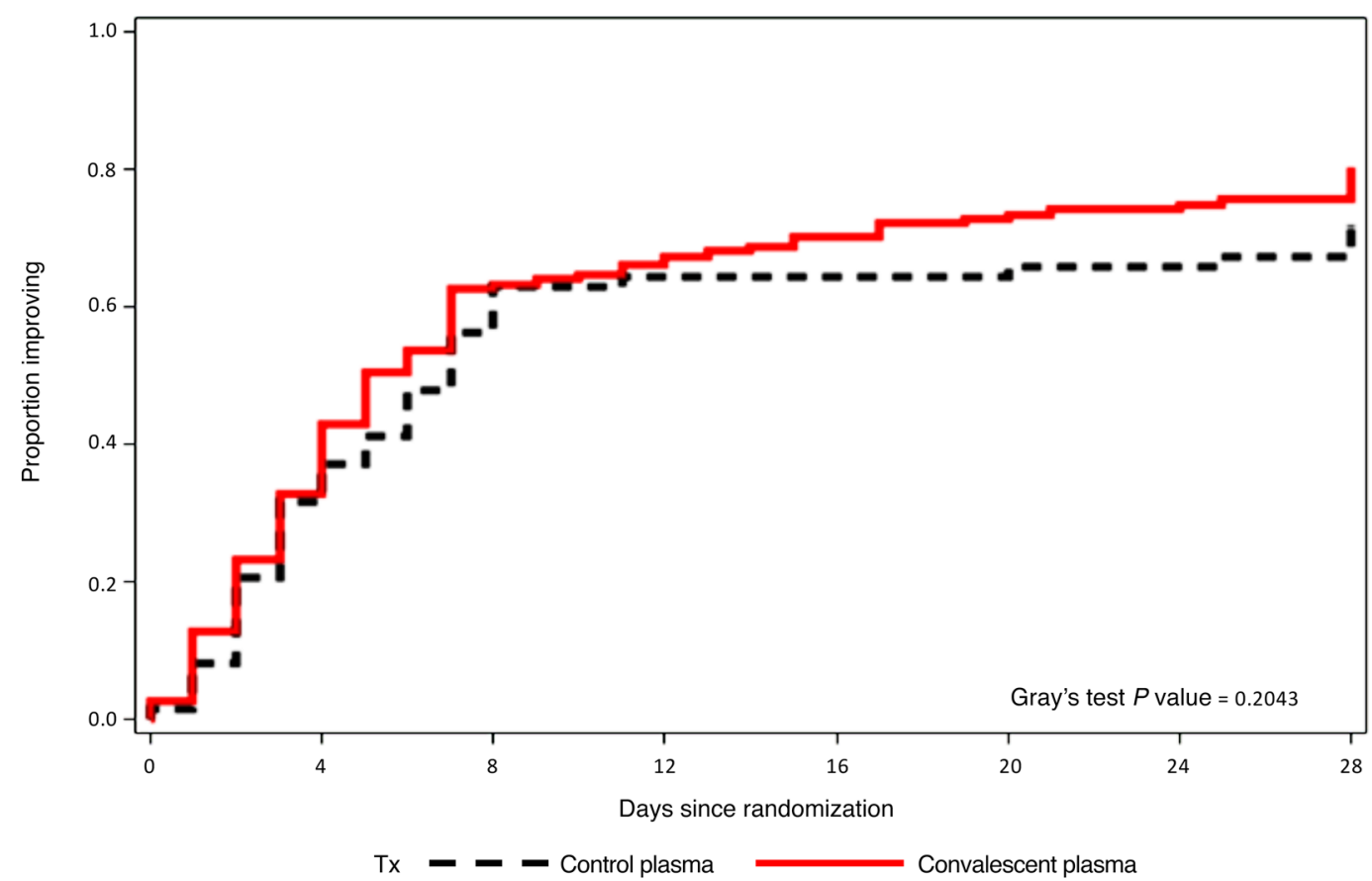

Time since randomization (days)

0

4

8

12

16

20

24

At risk

49

19

15

12

10

Control

Improving

27

47

47

48

48

53

At risk

147

96

46

39

30

25

21

16

Convalescent

plasma

Improving

63

93

99

103

108

110

117

Figure 3. Time-to-clinical improvement with death considered a competing risk, stratified by treatment group.

er control plasma units could have contained anti-coronavirus antibodies. Third, the median duration of symptoms at baseline was 9 days; earlier administration of high-titer convalescent plasma may have a higher potential for benefit (15). Fourth, supportive care was not standardized across study sites. However, we observed no significant differences in outcomes stratified by country.

In conclusion, although use of convalescent plasma was not associated with improved clinical status at 28 days, mortality at this time point was significantly reduced. This result should be interpreted with caution until full results from larger inpatient trials adequately powered to detect differences in mortality are available.

\section{Methods}

Study design. This was an investigator-initiated, randomized, double-blind, controlled trial to evaluate the efficacy and safety of convalescent plasma among adults hospitalized with severe COVID-19. The trial was conducted at 5 sites in New York City (USA) and Rio de Janeiro (Brazil) and was coordinated by Columbia University. Study sites included 2 hospitals affiliated with New York-Presbyterian Hospital/Columbia University Irving Medical Center (CUIMC) in northern Manhattan
(Milstein and Allen Hospitals) and 3 sites in Rio de Janeiro (Instituto Nacional de Infectologia Evandro Chagas, Hospital Federal dos Servidores do Estado, and Hospital Geral de Nova lguaçu). Participants were enrolled at CUIMC beginning April 21, 2020, and at the 3 clinical sites in Rio de Janeiro beginning August 15, 2020. The trial protocol was previously published and is available as supplementary material (21).

Participants. Eligible participants were hospitalized patients aged 18 years or older with evidence of SARS-CoV-2 infection by PCR of nasopharyngeal, oropharyngeal swab or tracheal aspirate sample within 14 days of randomization, with infiltrates on chest imaging and oxygen saturation less than or equal to $94 \%$ on room air or requirement for supplemental oxygen (including noninvasive positive pressure ventilation or high flow supplemental oxygen), IMV, or extracorporeal membrane oxygenation (ECMO) at the time of screening. Exclusion criteria included participation in another clinical trial of antiviral agent(s) for COVID-19; receipt of any antiviral agent with possible activity against SARS-CoV-2 within 24 hours of randomization; duration of IMV or ECMO 5 days or longer at time of screening; severe multiorgan failure; and a history of prior reactions to transfusion blood products. Following the U.S. Food and Drug Administration 


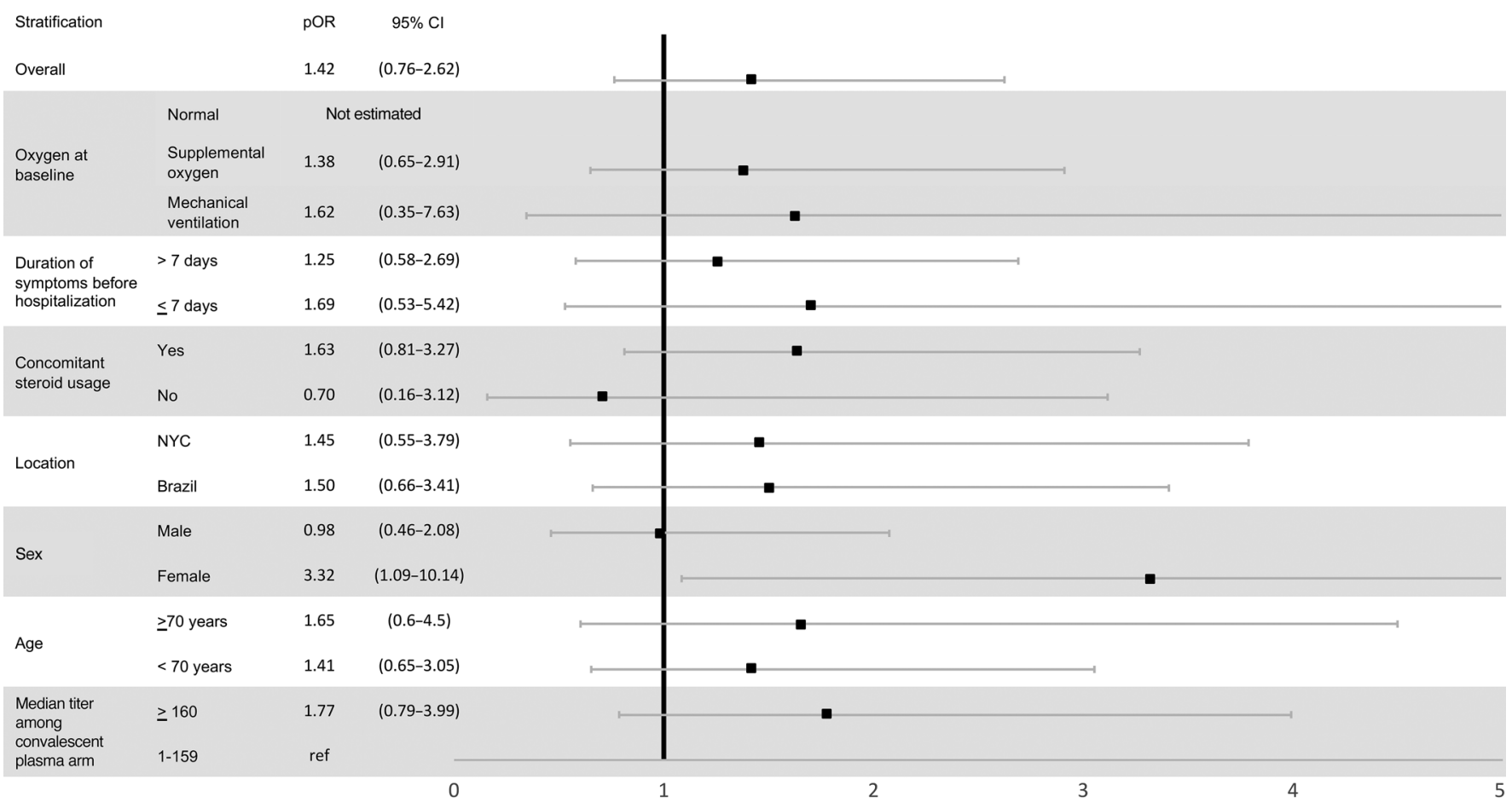

Figure 4. Subgroup analyses of primary outcome of clinical status at 28 days, adjusted for age and sex. pOR, proportional odds ratio.

Emergency Use Authorization on May 1, 2020 (22), concomitant use of remdesivir was permitted. The use of other treatments, including corticosteroids, was at the discretion of treating clinicians, and supportive care was provided according to standards at each site.

Procedures. Convalescent plasma used at all study sites was collected by the New York Blood Center from patients who had recovered from laboratory-confirmed COVID-19, provided informed consent, had a minimum anti-SARS-CoV-2 total IgG antibody titer of at least 1:400 by quantitative enzyme linked immunosorbent assay against the Spike protein (23), were at least 14 days asymptomatic following resolution of COVID-19, and had a negative PCR test for SARS-CoV-2 from a nasopharyngeal swab. Control plasma consisted of oldest available plasma at each study site without prior testing for anti-SARSCoV-2 antibodies. All control plasma was collected prior to January 1, 2020, in Rio de Janeiro and February 20, 2020, in New York City. For all participants who received their treatment assignment, a single unit of plasma ( 200-250 milliliters) was transfused over approximately 2 hours. Titers of neutralizing anti-SARS-CoV- 2 antibody were measured in convalescent plasma units post hoc. Neutralization titer was determined with a SARS-CoV-2 viral neutralization assay, which measured inhibition of virus growth after exposure to serial plasma dilutions using quantitative real-time reverse transcription-PCR (qRT-PCR). Further details are described in the protocol (21) and supplement. Given concern for emerging viral variants, we performed genomic sequencing of SARS-CoV-2 on nasopharyngeal swab samples from a subset of patients enrolled in Brazil. Sequences were mapped to the SARS-CoV-2 reference genome (sequence NC_045512) in NCBI. Additional methodological details are included in the supplement.

Randomization and blinding. Enrolled participants were randomized in a 2:1 ratio to receive either convalescent plasma or control plasma using a web-based randomization platform; treatment assign- ments were generated using randomly permuted blocks of different sizes. Randomization was stratified by site but not by severity of illness. Participants were transfused within 48 hours of randomization. The clinical teams directly managing patients and the trial clinicians who adjudicated clinical status and determined 28-day outcomes were blinded to treatment allocation. The hospital blood bank at each site and the clinical research teams who completed case record forms and performed other study specific procedures were not blinded; this was done to prevent errors in treatment allocation.

Outcomes. The primary outcome was clinical status at day 28 following randomization, measured using an ordinal scale based on that recommended by the World Health Organization (24), as follows: 1, not hospitalized with resumption of normal activities; 2, not hospitalized, but unable to resume normal activities; 3 , hospitalized, not requiring supplemental oxygen; 4, hospitalized, requiring supplemental oxygen; 5 , hospitalized, requiring high-flow oxygen therapy or noninvasive mechanical ventilation; 6, hospitalized, requiring ECMO, IMV, or both; 7, death. Since distinguishing between clinical status 1 and 2 on the ordinal scale was difficult in participants discharged from hospital, these 2 scores were combined, and a 6-point ordinal scale was used for all analyses of the primary outcome. Prespecified secondary outcomes included time-to-clinical improvement (defined as improvement in at least 1 point from baseline on the ordinal scale or alive at discharge from hospital, whichever came first), in-hospital mortality, 28-day mortality, time to discontinuation of supplemental oxygen, time to hospital discharge, and serious and grade 3 and 4 adverse events.

The initial primary outcome was time to clinical improvement. However, it became clear that this primary outcome would not reflect instances when patients' clinical status subsequently worsened after improvement. Thus, the primary outcome of the study was amended to clinical status at day 28 , and time to clinical improvement became 
a secondary outcome. This change was made on August 8, 2020 (at which point $31 \%$ [70/223] of the trial population was enrolled) without any knowledge of outcome data, and the protocol was updated accordingly with approval of the data safety and monitoring board (21).

Clinical status and adverse events were assessed daily during hospitalization through review of medical records and/or in-person visits. For participants discharged prior to day 28, clinical status and adverse events were determined via telephone and/or in-person visits. In patients who were discharged from hospital alive and not reachable for day 28 assessment, the last available clinical status was carried forward for the primary analysis, and sensitivity analyses were performed to account for potential bias due to loss-to-follow-up.

Statistics. The trial was analyzed by comparing patients randomized to convalescent plasma versus control plasma, with patients randomized to control plasma serving as the reference group. The primary outcome was analyzed using a 1-sided Mann-Whitney test for an alternative hypothesis favoring the convalescent plasma arm (a "go" decision in this phase 2 trial). To assess the magnitude of clinical effects, an OR for improved clinical status on the modified ordinal scale was estimated under the proportional odds model. An OR greater than 1.0 indicated improved clinical status among patients randomized to convalescent plasma versus control plasma. Post hoc subgroup analyses for odds of clinical improvement and mortality were performed according to study country, age, sex, concomitant treatment with corticosteroids, and by titers of neutralizing anti-SARS-CoV-2 antibody in infused convalescent plasma units as reported in the supplement.

Prespecified subgroups in analyses of the primary outcome were defined according to level of respiratory support at randomization (no supplemental oxygen, supplemental oxygen [including high-flow oxygen therapy and noninvasive ventilation], IMV or ECMO) and symptom duration at randomization ( $\leq 7$ days, $>7$ days) (21). Post hoc subgroup analyses were performed according to study country, age, sex, concomitant treatment with corticosteroids, and by titers of neutralizing anti-SARS-CoV-2 antibody in infused convalescent plasma units.

For the initial primary outcome of time to clinical improvement, the intended sample size was 129 participants. However, after the primary outcome was amended, the sample size was recalculated based on blinded pooled data of day 28 outcomes from an interim analysis by the data safety and monitoring board (July 2, 2020) and an OR of 1.7 under a proportional odds assumption. With a 2:1 randomization ratio and a total sample size of 219 participants (146 in the convalescent plasma arm versus 73 in the control arm), we determined that a 1-sided Mann-Whitney test at a level of $15 \%$ would have $82 \%$ power to detect an OR 1.7 . At the time the primary outcome was amended, a recent trial of remdesivir reported an OR 1.50 with 95\% CI of 1.18-1.91, which overlapped with our assumed OR (25).
Between-group differences are reported using point estimates (OR or hazard ratio [HR]), with 95\% CIs and $P$ values. The $P$ value for the Mann-Whitney test in the primary outcome analysis ("go vs. "no-go" decision) is 1-sided. All other $P$ values, including those associated with point estimates, are 2-sided and without adjustment for multiple comparisons. Analyses were performed using SAS, version 9.4 (SAS Institute).

Study approval. The trial was conducted in accordance with good clinical practice guidelines, the Declaration of Helsinki, and the Brazilian National Ethics Committee Resolution 466/12. Written informed consent was obtained from all participants or from their legally authorized representative. The study protocol, definition of outcomes, and other relevant materials have been published previously (21). The trial protocol was approved by institutional review boards at CUIMC and at each site in Rio de Janeiro (21) and is registered at ClinicalTrials.gov (identifier: NCT04359810).

\section{Author contributions}

MRO and WIL conceived the study and led protocol development. MRO, MJC, JEJ, NMP, AE, YKC, and WIL contributed to study design. MRO, BG, MJC, CME, NMP, MRL, YKC, EJ, JHP, MPD, SWC, DA, KNR, LCS, AV, VGV, DES, BJM, SDJ, and WIL contributed to data acquisition, analysis, and/or interpretation. NM, LVC, TB, and WIL performed and interpreted neutralization assay experiments. EAH, ZCB, SLS, EFS, FDZ, FLC, KEH, SAF, JS, BAS, WHWL, SNW, and BS contributed to convalescent plasma collection, qualification, and release. SEB, AK, AW, and NMP coordinated study activities. MRL and YKC performed statistical analyses. MRO, MJC, MRL, YKC, VG, and WIL wrote the manuscript. All authors contributed to critical revision of the manuscript.

\section{Acknowledgments}

This trial was funded by unrestricted grants from Amazon, the Skoll Foundation, and the Chau Hoi Shuen Foundation to Columbia University. The authors would like to thank the patients who participated in the study and their families as well as the members of the data safety and monitoring board (Neil W. Schluger, Scott M. Hammer, Deborah Donnell).

Address correspondence to: Max R. O'Donnell, Division of Pulmonary, Allergy, and Critical Care Medicine, Columbia University Irving Medical Center, 622 West 168th Street, PH 8E-101, New York, New York 10032, USA. Phone: 212.305.5794; Email: mo2130@columbia.edu.
1. Dong E, et al. An interactive web-based dashboard to track COVID-19 in real time. Lancet Infect Dis. 2020;20(5):533-534.

2. Wu Z, McGoogan JM. Characteristics of and important lessons from the coronavirus disease 2019 (COVID-19) outbreak in China: summary of a report of 72314 cases from the Chinese Center for Disease Control and Prevention. JAMA. 2020;323(13):1239-1242.

3. U.S. Centers for Disease Control and Prevention. COVIDView: A Weekly Surveillance Summary of U.S. COVID-19 Activity. https://www.cdc.gov/ coronavirus/2019-ncov/covid-data/covidview/ index.html. Updated May 7, 2021. Accessed May 10, 2021.

4. Cummings MJ, et al. Epidemiology, clinical course, and outcomes of critically ill adults with COVID19 in New York City: a prospective cohort study. Lancet. 2020;395(10239):1763-1770.

5. Casadevall A, Pirofski LA. The convalescent sera option for containing COVID-19. J Clin Invest. 2020;130(4):1545-1548.

6. Mair-Jenkins J, et al. The effectiveness of convalescent plasma and hyperimmune immunoglobulin for the treatment of severe acute respiratory infections of viral etiology: a systematic review and exploratory meta-analysis. JInfect Dis. 2015;211(1):80-90.

7. Liu STH, et al. Convalescent plasma treatment of severe COVID-19: a propensity score-matched control study. Nat Med. 2020;26(11):1708-1713.

8. Duan K, et al. Effectiveness of convalescent plasma therapy in severe COVID-19 patients. Proc Natl Acad Sci U S A. 2020;117(17):9490-9496.

9. Joyner MJ, et al. Safety update: COVID-19 convalescent plasma in 20,000 hospitalized patients. Mayo Clin Proc. 2020;95(9):1888-1897. 
10. Joyner MJ, et al. Convalescent plasma antibody levels and the risk of death from Covid-19. N Engl JMed. 2021;384(11):1015-1027.

11. Li L, et al. Effect of convalescent plasma therapy on time to clinical improvement in patients with severe and life-threatening COVID-19: a randomized clinical trial. JAMA. 2020;324(5):460-470.

12. Agarwal A, et al. Convalescent plasma in the management of moderate covid-19 in adults in India: open label phase II multicentre randomised controlled trial (PLACID Trial). BMJ. 2020;371:m3939.

13. Horby PW, et al. Convalescent plasma in patients admitted to hospital with COVID-19 (RECOVERY): a randomised, controlled, open-label, platform trial [preprint]. https://doi.org/10.11 01/2021.03.09.21252736. Posted on medRxiv March 10, 2021.

14. Simonovich VA, et al. A randomized trial of convalescent plasma in Covid-19 severe pneumonia. N Engl JMed.2021;384(7):619-629.

15. Libster R, et al. Early high-titer plasma therapy to prevent severe Covid-19 in older adults. $N$ Engl J Med.2021;384(7):610-618.
16. U.S. Centers for Disease Control and Prevention. Emerging SARS-CoV-2 variants. https:// www.cdc.gov/coronavirus/2019-ncov/more/ science-and-research/scientific-brief-emerging-variants.html. Updated January 28, 2021. Accessed May 10, 2021.

17. Group A-TL-CS, et al. A neutralizing monoclonal antibody for hospitalized patients with Covid-19. N EnglJMed. 2021;384(10):905-914.

18. U.S. Food and Drug Administration. Development of Monoclonal Antibody Products Targeting SARSCoV-2, Including Addressing the Impact of Emerging Variants During the COVID-19 Public Health Emergency. https://www.fda.gov/regulatoryinformation/search-fda-guidance-documents/ development-monoclonal-antibody-productstargeting-sars-cov-2-including-addressing-impactemerging. Accessed May 10, 2021.

19. Sanfilippo F, et al. COVID-19, Hypercoagulability, and cautiousness with convalescent plasma. Am J Respir Crit Care Med. 2021;203(2):257-258.

20. Jimenez D, et al. Incidence of VTE and bleeding among hospitalized patients with coronavirus disease 2019: a systematic review and meta-analysis. Chest. 2021;159(3):1182-1196.

21. Eckhardt CM, et al. Evaluating the efficacy and safety of human anti-SARS-CoV-2 convalescent plasma in severely ill adults with COVID-19: a structured summary of a study protocol for a randomized controlled trial. Trials. 2020;21(1):499.

22. U.S. Food and Drug Administration. Remdesivir emergency use authorization. https://www.fda.gov/ media/137564/download. Accessed May 10, 2021.

23. Weisberg SP, et al. Distinct antibody responses to SARS-CoV-2 in children and adults across the COVID-19 clinical spectrum. Nat Immunol. 2021;22(1):25-31.

24. World Health Organization. WHO R\&D Blueprint - COVID-19 Therapeutic Trial Synopsis. https://www.who.int/blueprint/prioritydiseases/key-action/COVID-19_Treatment_ Trial_Design_Master_Protocol_synopsis_ Final_18022020.pdf. Accessed May 10, 2021.

25. Beigel JH, et al. Remdesivir for the treatment of Covid-19 - final report. $N$ Engl J Med. 2020;383(19):1813-1826. 